\title{
A Two-Level Inspection Model With Technological Insertions
}

\author{
Wenbin Wang, Matthew J. Carr, Tommy W. S. Chow, Senior Member, IEEE, and Michael G. Pecht, Fellow, IEEE
}

\begin{abstract}
This paper presents a model for optimal asset maintenance inspection services. The model is designed to support through-life service in the form of multiple nested inspections and maintenance to meet defined asset availability and capability requirements, as well as achieving successful through-life technology insertions. The inspections and maintenance activities are assumed to be performed at more than one level, but nested and aimed at different types of defects or subsystems over a fixed period of time (the designed asset life). This practice is common in many industries, particularly in the defense industry. The impact of technological insertions is reflected through changes in the failure behavior of the asset. We use the delay time concept to model the failure mechanism of the asset, and the arrivals of defects are assumed to follow Poisson processes. The decision variables are the inspection intervals, while the objective function can be expressed in terms of cost, downtime, or reliability. The model is demonstrated through a numerical example. The model can be used for optimizing two-level inspection intervals with technological insertions.
\end{abstract}

Index Terms-Delay time, inspection, maintenance, technological insertions.

\section{ACRONYM}

DP Dynamic Programming

HPP Homogenous Poisson process

\section{NOTATION}

$T \quad$ Planned service life

$k \quad$ Defect type

$\lambda_{k} \quad$ The rate of type $k$ defect arrival

$h \quad$ The delay time of a defect

Manuscript received December 12, 2009; revised February 08, 2011 and August 15, 2011; accepted October 26, 2011. Date of publication February 23, 2012; date of current version May 28, 2012. This work was partially supported by a Grant from the Research Grants Council of the Hong Kong Special Administrative Region, China (CityU8/CRF/09). The research reported here was also partially supported by the NSFC China under Grant 71071097. Associate Editor: L. Walls.

W. Wang is with Dongling School of Economics and Management, University of Science and Technology, Beijing, China.

M. J. Carr is with School of Medicine, University of Manchester, Manchester, U.K.

T. W. S. Chow is with Department of Electronic Engineering, City University of Hong Kong, Hong Kong.

M. G. Pecht is with CALCE Electronics Products and Systems Centre, University of Maryland, College Park, MD, USA, and also PHM Centre, City University of Hong Kong, Hong Kong.

Color versions of one or more of the figures in this paper are available online at http://ieeexplore.ieee.org.

Digital Object Identifier 10.1109/TR.2012.2183911
$f_{k}(h)$
The type $k$ defect delay time distribution
$U_{k f}$
The average loss measure for a type $k$ failure, where $U$ could be downtime or cost
$U_{k s} \quad$ The average loss measure for a type $k$ inspection
$U_{k d} \quad$ The average loss measure for a type $k$ defect removal at a type $k$ inspection
$\mathrm{E}\left[N_{k f}(t)\right]$ The expected number of type $k$ failures over an interval $(0, t]$
$\mathrm{E}\left[N_{k d}(t)\right]$ The expected number of type $k$ defect removals at a type $k$ inspection after an interval of duration $t$
$\mathrm{E}[U(\bullet)] \quad$ Expected loss over $T$ with $\bullet$ as the decision variables
$\mathrm{E}[V(\bullet)] \quad$ Expected loss per unit time with $\bullet$ as the decision variables

The term loss can refer to any penalty of a measurable nature that is related to the performance of the asset, such as cost or downtime. The sub-script $k$ is omitted in the above notation when a single type of defect and inspection is considered.

\section{INTRODUCTION}

$\mathbf{T}$ IME-BASED inspection and maintenance is still one of the dominant maintenance policies used in industry for certain types of assets that cannot be condition-monitored or maintained on a predictive basis [1]. For complex engineering assets such as aircraft, commercial and military vehicles, and infrastructures such as 1rail tracks and utility networks, timebased inspection and maintenance policies can improve performance, and increase the reliability and capability of the assets concerned, [2], [3]. It can also provide the benefit of easy planning. On the other hand, due to advances in technology and production, longer product life cycles and increased asset availability are increasingly common requirements within the capital-intensive industries (such as offshore applications, defense, and aviation), and represent important considerations for the development of maintenance service plans, and technological upgrading activities. Improving the provision of maintenance to incorporate optimal inspection intervals throughout the proposed life of an asset should theoretically result in a reduction in the cost of running the asset, and ultimately a contribution to the bottom line business of the asset owners and users [4].

For complex asset systems with many subsystems and components, the planned inspection and maintenance will normally 
be undertaken at different intervals, levels, and depths. Typical practice involves a routine inspection on a more frequent basis for some subsystems or components that may be subject to more frequent failures, followed by a longer interval applied to the system as a whole. An example of such maintenance service practice can be seen in aircraft maintenance where up to four different maintenance intervals may be in place [2]. These different intervals are usually nested, as up-level maintenance with a longer interval will include the content of lower levels of maintenance with shorter intervals [5], [6]. We show later that a multiple inspection scheme at different intervals is better than a single inspection for the whole system if failures can be captured separately.

Capital assets normally have longer lifecycles, and longer lifecycles mean that the likelihood of technological obsolescence is substantially increased. This effect results in the necessity for improved maintenance, repairs, and spare parts planning to achieve the goal of system capability enhancement. To provide a competitive edge within the market, satisfying the availability demands of customers is a necessity. Doing such requires a cohesive, cost-effective maintenance framework with the provision for technological insertion planning. Technological insertions are considered in many managerial papers in a qualitative manner [7]-[9]. These studies mainly address the framework, concepts, components, and dimensions of such insertions. However, regardless of the particular application, justification for the technological insertions is required in the form of a cost benefit analysis that should demonstrate the expected contribution with regard to the minimization of associated risks and costs.

Technological insertions must have an impact upon the failure behavior of the asset, and therefore on the associated inspection policy as well [10]. There is currently very little underlying academic theory and few methodological options available in the literature to assist in the consideration of time-based multiple inspections with technological insertions over a finite time horizon. Most of the literature on inspection modeling focuses on a single type of inspection interval [1], [11]. The difficulty arises from the changing nature of the failure mechanisms of the asset due to technological insertions, where a constant failure rate assumption cannot be adopted, and the multiple inspection intervals over a finite time horizon are needed. Wang [5] studied a similar problem of nested inspections using sequential optimization with final replacement, but did not consider technological insertions. Hosseini et al. [12] studied two types of repairs after an inspection using generalized stochastic Petri Nets. Li and Pham [13] studied an inspection-maintenance model in the context of multiple competing failure modes. Cui et al. [14] presented a model for sequential inspection strategies, but the focus was on the case when there are multiple systems inspected at the same but discrete time points. Though the above studies [12]-[14] have some relevance to this paper, none have considered the problem of incorporating more than one type of inspection or technological insertion activity. In a recent paper, Wang [6] considered two types of inspections and repairs. It is the work most closely related to this paper, but the model was established under the assumption of an infinite horizon, and again no changing behavior of the asset influenced by technological insertions was considered. However, we have to point out that obtaining an estimate of the impact of technological insertions on the failure behavior of an asset is a challenging task that will often be application-specific.

The model developed in this paper differs from the previous models in two aspects. First, as discussed, the time horizon is finite, and defined as the designed or anticipated asset life. Secondly, due to the provision for technological insertions, the inspection interval may not be constant, and therefore the consideration of a dynamic inspection policy is required. The new contributions of this paper are therefore as follows. 1) We prove that there is a condition in which an optimal inspection interval exists. 2) We also show that a multiple inspection interval scheme is better than a single inspection interval scheme if defect arrivals and failures can be modeled differently. 3) We propose an algorithm for the optimal dynamic determination of the sequential inspection intervals using dynamic programming, which is superior to constant inspection interval policies. The developed model can facilitate the through-life process of technological insertions in the form of a cost-benefit analysis, and jointly optimize the inspection intervals associated with the asset over a finite time horizon, defined as the asset life. The model can also provide a sensitivity analysis of the impact of technological insertions and inspection scheduling on asset performance in terms of cost or any other criterion of interest. Though the model developed is for an asset with two types of inspections, the ideas and principles can be generalized for systems with more types of inspections.

We consider a model of two-type inspection services and maintenance where upgrading activities and technological insertions are regarded as part of the maintenance planning process, and capability enhancement trade-off decisions are readily incorporated. Initially, we discuss the infinite horizon modeling scenario as an introduction before moving on to discuss the finite horizon model. The model looks at how different lines or levels of maintenance service can be optimized over the lifecycle of an asset to provide through-life support while balancing the competing objectives of capability and affordability. The model can be easily tailored to a given application, and can be trained using either observed failures or subjective expert information within a Bayesian framework. This model is targeted at answering the question of when to perform the maintenance tasks while also investigating the impact of technological insertions. Necessary proofs are given, and we demonstrate the methodology using a numerical example at the end of this paper. For practical applications, the models can be easily implemented into a computerized maintenance planning package as a module where the complicated optimization process can be done automatically.

\section{Modeling Assumptions}

1) A multi-component complex system.

2) All failures follow a two-stage failure process as defined by the well-known delay time concept [15]: from new to the initial point of a hidden defect, then from this initial point to an eventual failure caused by the defect if it is not attended to. 
3) If an inspection service is performed during the second stage before the failure, then the defect may be rectified by either a repair or replacement of the same or an upgraded part.

4) There are two types of defects: minor, and major.

5) Minor defect arrivals follow a homogeneous Poisson process (HPP) with a constant average rate.

6) Major defect arrivals follow a stepwise HPP with a constant average rate during the major inspection interval, but changing at the time of the major inspection. (This is due to technological insertions; and if such insertions are not there, then no change occurs.)

7) The downtimes caused by failures and inspections are ignored when calculating the expected number of failures and defect identifications because they are usually small compared with the inspection intervals. The downtimes are usually measured in minutes, but the inspection intervals may be measured in weeks or months. However, these downtimes will be included when formulating the downtime model.

8) Once a defect has arisen, it follows a delay time $h$ with a probability density function.

9) All inspections are perfect in that they can identify the intended defect.

10) All defects identified at inspection are rectified, but may require additional downtime and costs.

The delay time concept and associated techniques for inspection modeling have been reported in many papers over the last 25 years [10], [15]-[19]. The delay time, as defined in assumption 2, provides a window for inspection and repair, and as such, the relationship between failures and the inspection process can be captured. Applications of the delay time concept and the associated modeling techniques can be seen for various industrial assets [16], [17], [20]-[23].

Fig. 1 illustrates a typical defect arrival, failure, and planned inspection process for an inspection interval $t$, where defects that are currently present in the system are identified and removed. The circles represent the initiation of random defects, and the dots represent failures caused by these defects if no maintenance intervention takes place. The arc linking a circle and a dot is the delay time of the defect. Obviously, because of planned inspection interventions (assuming that identified defects will be removed), some defects are identified at inspections and rectified. This approach reduces the number of failures from the original 7 to 3. Clearly, the intervals of such inspections are important, because more frequent inspections will identify and remove more defects, and consequently reduce the cost of failures, but will result in an increased cost for inspections. Modeling is necessary to balance the trade-off between these two costs.

\section{Delay TIME Modeling}

The delay time concept itself is simple; the failure process of a system is defined as a two-stage process. The first stage is the usual operating stage from new until the initiation of a hidden defect. The second stage is defined as the failure delay time from

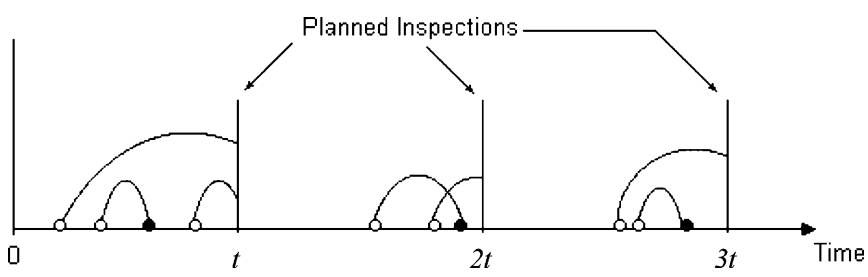

Fig. 1. The defect arrival, failure, and inspection removal process.

the point of defect initiation until failure. It is the existence of such a failure delay time that provides the opportunity for preventive maintenance to be carried out to remove or rectify the identified defects before failures occur. With appropriate modeling of the duration of these two stages, an inspection interval can be identified that optimizes the criterion function of interest.

If there is only one type of defect, given the average defect arrival rate $\lambda$ and the delay time distribution $f(h)$, the relationship between the inspection cycle length $t$, and the expected loss per unit time, can be established directly using renewal reward theory [19]. Consider an interval $[u, u+\delta u)$ for $0 \leq u<t$, and $\delta u \rightarrow 0^{+}$. The expected number of defect arrivals within $[u, u+\delta u)$ is $\lambda \delta u$; and, for a cycle of length $t$, the expected number of failures resulting from the defects is $\lambda F(t-u) \delta u$, where $F(h)$ is the cumulative delay time distribution. Integrating $u$ from 0 to $t$, and after some manipulation, we have the expected number of failures over interval $[0, t)[19]$ as

$$
\mathrm{E}\left[N_{f}(t)\right]=\int_{0}^{t} \lambda F(h) d h .
$$

Differentiating $\mathrm{E}\left[N_{f}(t)\right]$ with respect to $t$ gives the rate of failure at time $t$ as

$$
v(t)=\lambda F(t)
$$

The failure process conforms to a non-homogenous Poisson process

$$
N_{f}(t) \sim \operatorname{Po}\left\{\mathrm{E}\left[N_{f}(t)\right]\right\}
$$

where

$$
\mathrm{E}\left[N_{f}(t)\right]=\int_{0}^{t} v(u) d u .
$$

The expected number of defects detected and removed at an inspection after an operational interval of duration $t$ is

$$
\mathrm{E}\left[N_{d}(t)\right]=\int_{0}^{t} \lambda(1-F(h)) d h .
$$

A number of modifications to the basic formulation have been considered in the literature including non-constant defect arrival rates $\lambda(u)$, and imperfect inspection processes [15].

\section{INFINITE HORIZON INSPECTION MODELS}

Initially, we investigate infinite horizon planning scenarios before moving to the case of a finite horizon. This infinite horizon assumption will serve as an introduction because it is relatively easy to model, and most past delay time modeling 
research used this assumption. In the first case, we explore the scheduling of system wide inspections where the behavior of the system is aggregated and modeled collectively. In the second case, we explore the potential benefits of multiple inspection intervals for different defect types, categorized here as minor and major defects.

\section{A. A Single Inspection Interval}

Firstly, we explore the case where the aggregated behavior of the system is analyzed and optimized. Defining $V(t)$ as the loss per unit time for an interval between inspections of duration $t$, we have the expectation

$$
\mathrm{E}[V(t)]=\left\{\mathrm{E}\left[N_{f}(t)\right] U_{f}+U_{s}+\mathrm{E}\left[N_{d}(t)\right] U_{d}\right\} / t
$$

where $U_{f}$ is the average loss associated with a failure, and $U_{s}$ is the average loss associated with a scheduled inspection with $U_{d}$ as the average loss associated with a preventive defect repair during an inspection. Note that, in this case, the loss measures are averaged out across the system as a whole. Using (1) and (3) gives

$\mathrm{E}[V(t)]=\left\{U_{f} \int_{0}^{t} \lambda F(h) d h+U_{s}+U_{d} \int_{0}^{t} \lambda(1-F(h)) d h\right\}_{(5)} / t$

where $\lambda$ is the system-wide defect arrival rate, and $F(h)$ is the cumulative delay time distribution of all defects.

Lemma 1: The necessary and sufficient condition for an optimal inspection interval $t^{*}$ is

$$
\lambda\left(t * F(t *)-\int_{0}^{t *} F(h) d h\right)=U_{s} /\left(U_{f}-U_{d}\right),
$$

which is obtained via the differentiation of $\mathrm{E}[V(t)]$ with respect to $t$, and setting it to 0 . It is clear that we must have the condition $U_{f}>U_{d}$ as the left hand side of (6) is always positive for any probability density function (pdf), and corresponding cumulative density function (cdf). Clearly, if $U_{f} \leq U_{d}$, then there is no need for inspections.

Lemma 2: The optimal solution $t^{*}$ is unique if

$$
\lambda E(H)>U_{s} /\left(U_{f}-U_{d}\right)
$$

where $E(H)$ is the expected delay time.

Proof: From (6), when $t=0$, we have the LHS of (6) equal to zero. When $t$ increases, the LHS of (6) increases monotonically to a constant $\lambda E(H)$. This result can be easily shown as

$$
\begin{aligned}
& \lim _{t \rightarrow \infty}\left(F(t) t-\int_{0}^{t} F(x) d x\right) \\
& =\operatorname{Lim}_{t \rightarrow \infty}\left(F(t) t-\int_{0}^{t}(1-S(h)) d h\right) \\
& =\int_{0}^{\infty} S(h) d h=E(H) .
\end{aligned}
$$

Then if (7) is satisfied, there must be a value of $t^{*}$ that optimizes (5).

Example 1: A Single Inspection Interval: For this example, we use an average system-wide defect arrival rate $\lambda=0.25$, and an exponential delay time distribution; $f(h)=\alpha \exp (-\alpha h)$ with parameter $\alpha=0.05$. The average loss measures are taken

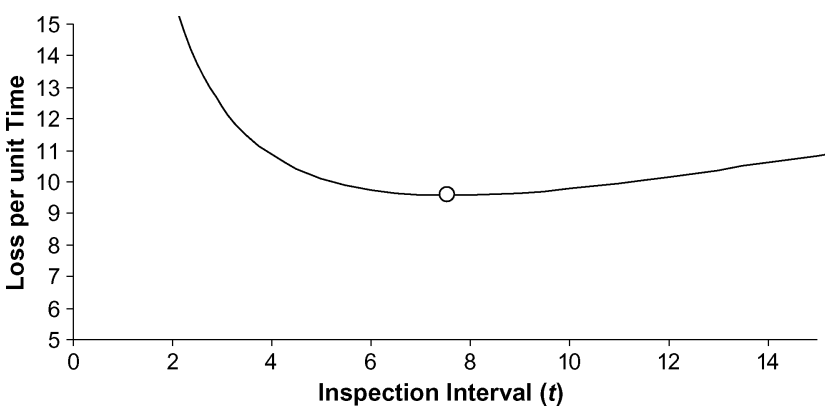

Fig. 2. Illustration of the expected loss per unit time against the inspection interval.

to be $U_{f}=100$ for a failure of the system, $U_{s}=25$ for an inspection of the system, and $U_{d}=10$ for an inspection-based defect repair.

From (1), (3), and (4), we have the expected number of failures

$$
\begin{aligned}
\mathrm{E}\left[N_{f}(t)\right] & =\int_{0}^{t} \lambda F(h) d h \\
& =\int_{0}^{t} \lambda\left(1-e^{-\alpha h}\right) d h=\lambda t-(\lambda / \alpha)\left(1-e^{-\alpha t}\right),
\end{aligned}
$$

and the expected number of defect repairs at inspection

$$
\begin{aligned}
\mathrm{E}\left[N_{d}(t)\right] & =\int_{0}^{t} \lambda(1-F(h)) d h \\
& =\int_{0}^{t} \lambda e^{-\alpha h} d h=(\lambda / \alpha)\left(1-e^{-\alpha t}\right)
\end{aligned}
$$

giving the expected loss per unit time for an inspection interval of duration $t$ :

$$
\mathrm{E}[V(t)]=\left\{\lambda t U_{f}-\left(U_{f}-U_{d}\right)(\lambda / \alpha)\left(1-e^{-\alpha t}\right)+U_{s}\right\} / t .
$$

Using (13), Fig. 2 illustrates the expected loss per unit time against the inspection interval.

Minimizing the expected loss per unit time with respect to the inspection interval $t$, the optimal inspection interval for this case is found to be $t^{*}=7.545$. This optimal interval can be verified using (6), as illustrated in Fig. 3 where the left hand side of 6) is defined as $g(t)$.

Using (7), we can verify that the optimal inspection interval is unique:

$$
\lambda E(H)=\lambda / \alpha=5>\frac{U_{s}}{U_{f}-U_{d}}=0.278 .
$$

This concludes the example.

\section{B. Multiple Inspection Intervals}

In this section, we address infinite horizon scheduling problems when different defect and failure types are identifiable. We consider the categorization of minor and major defects and failures, and the scheduling of corresponding minor and major inspection-based interventions with different loss measures. The different defect arrival rates are estimated $s$-independently as $\lambda_{1}$, and $\lambda_{2}$ for minor, and major defects, respectively. Similarly, the individual delay time distributions are specified as $f_{1}(h)$ and 


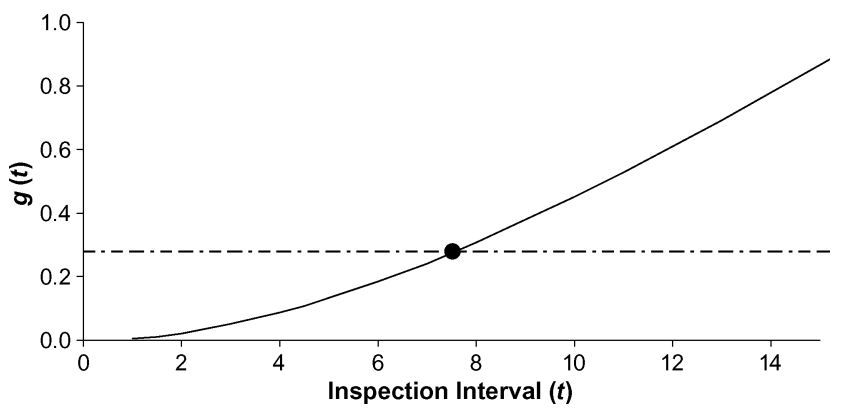

Fig. 3. Illustrating the evaluation of the optimal inspection interval $t^{*}=7.545$.

$f_{2}(h)$, and the average losses associated with failures $U_{f}$, inspections $U_{s}$, and preventive repairs $U_{d}$ are estimated $s$-independently for each defect type. We specify the following additional assumptions.

- There are two broad types of defects, and therefore failures: minor, and major.

- Minor defects can be identified and rectified by a minor inspection service, while major defects can only be identified and removed by a major inspection service. A major inspection service includes the content of a minor inspection service, and occurs at one of the minor inspection service interventions.

Common Inspection Model: Firstly, we consider the scenario where minor and major defects and failures are identifiable, but the minor and major inspections are undertaken on the same interval. This case is different from the model presented in the previous sub-section where the behavior of the system was aggregated. This model serves as a comparison for the subsequent model with different inspection intervals for the different defect types.

For a common inspection, on interval $t$, we have

$$
\begin{aligned}
& \mathrm{E}[V(t)]=\left\{\mathrm{E}\left[N_{1 f}(t)\right] U_{1 f}+\mathrm{E}\left[N_{1 d}(t)\right] U_{1 d}\right. \\
&\left.+\mathrm{E}\left[N_{2 f}(t)\right] U_{2 f}+\mathrm{E}\left[N_{2 d}(t)\right] U_{2 d}+U_{2 s}\right\} / t
\end{aligned}
$$

which can be written as

$$
\mathrm{E}[V(t)]=\left\{U_{2 s}+\sum_{k=1}^{2}\left(\mathrm{E}\left[N_{k f}(t)\right] U_{k f}+\mathrm{E}\left[N_{k d}(t)\right] U_{k d}\right)\right\} / t .
$$

As the two different failure processes evolve $s$-independently, using (1) and (3), the expectation can be expressed as

$$
\begin{array}{r}
\mathrm{E}[V(t)]=\left\{U_{2 s}+\sum_{k=1}^{2}\left(U_{k f} \lambda_{k} \int_{0}^{t} F_{k}(h) d h+U_{k d} \lambda_{k}\right.\right. \\
\left.\left.\times \int_{0}^{t}\left(1-F_{k}(h)\right) d h\right)\right\} / t .
\end{array}
$$

As such, the optimal inspection interval can be found as the solution to

$$
\sum_{k=1}^{2}\left(U_{k f}-U_{k d}\right) \lambda_{k}\left(t * F_{k}(t *)-\int_{0}^{t *} F_{k}(h) d h\right)=U_{2 s}
$$

with respect to $t^{*}$. However, unlike the single inspection case, the condition $U_{k f}>U_{k d}$ is not strictly required for both $k=1$ and 2. It can be shown in a manner similar to lemma 2 that a unique solution exists if $\sum_{k=1}^{2}\left(U_{k f}-U_{k d}\right) \lambda_{k} E\left(H_{k}\right)>U_{2 s}$, where $E\left(H_{k}\right)$ is the expected delay time for the $k$ th type of defects. Equation (11) can be solved by any mathematical software that can find the root of a function.

Multiple Inspection Model: Now we consider the multiple inspection case where minor and major inspections are scheduled. As discussed in the introduction to this section, major inspections contain all the activities of minor inspections. As such, major inspections are scheduled to occur at integer multiples of the minor inspection interval. With minor and major inspection intervals of duration $\Delta t$, and $m \Delta t$, respectively, we have

$$
\begin{aligned}
\mathrm{E}[V(\Delta t, m)]=\{ & m\left(\mathrm{E}\left[N_{1 f}(\Delta t)\right] U_{1 f}+\mathrm{E}\left[N_{1 d}(\Delta t)\right] U_{1 d}\right) \\
& +(m-1) U_{1 s}+\mathrm{E}\left[N_{2 f}(m \Delta t)\right] U_{2 f} \\
& \left.+U_{2 s}+\mathrm{E}\left[N_{2 d}(m \Delta t)\right] U_{2 d}\right\} / m \Delta t
\end{aligned}
$$

Naturally, when $m=1$, the model is the same as the common inspection model with $t=\Delta t$. This model is an optimization problem with decision variables $\Delta t$, and $m$. Similar to lemmas 1 and 2, we can show that for a given $m$, and $\Delta t$ respectively, unique solutions of $\partial E[V(\Delta t, m)] / \partial \Delta t=0$, and $\partial E[V(\Delta t, m)] / \partial m=0$ exist under certain conditions, so the optimal solution of (12) also exists under the same conditions in terms of $m$ and $\Delta t$.

Example 2: Common vs. Multiple Inspection Intervals: For this example, we assume minor, and major defect arrival rates of $\lambda_{1}=0.25$, and $\lambda_{2}=0.05$, respectively; and exponential delay time distributions $f_{1}(h)$ and $f_{2}(h)$ with parameters $\alpha_{1}=0.05$, and $\alpha_{2}=0.02$. The average loss values are taken to be $U_{1 f}=$ $15, U_{1 d}=2$, and $U_{1 s}=3$ for minor defects and inspections; and $U_{2 f}=50, U_{2 d}=15$, and $U_{2 s}=20$ for major defects and inspections.

The form of the expected number of failures, $\mathrm{E}\left[N_{k f}(t)\right]$, and the expected number of inspection repairs, $\mathrm{E}\left[N_{k d}(t)\right]$, are given by (1) and (3); and they are derived in the same manner as example 1 for both defect types $k=1$, and 2. Fig. 4 illustrates the expected loss per unit time for integer values of the minor inspection interval $\Delta t$ under the common and multiple inspection policies. Note that in Fig. 4, in the case of the common interval model, the major interval is the same as the minor interval, and in the multiple interval case, the major interval has been determined by optimizing $m$ for each integer value of the minor inspection interval. This work was done by enumerating $m$ at each $\Delta t$ until an optimal $m^{*}$ was found.

In the case of the common inspection model, the optimal interval is found to be 18.45 , giving an expected loss per unit time of 3.748. In the multiple inspection case, the optimal intervals are found by enumerating the minor interval $\Delta t$ over a finite range of values given that the optimal integer value of $m^{*}$ was already found for each $\Delta t$. For this example, we find the optimal minor inspection interval to be $\Delta t^{*}=6.679$, and $m^{*}=6$, giving an optimal major inspection interval of duration $6 \times 6.679=40.074$. Using the multiple inspection model, the expected loss per unit time is 3.156. Clearly, the multiple inspection policy is superior in this example. 


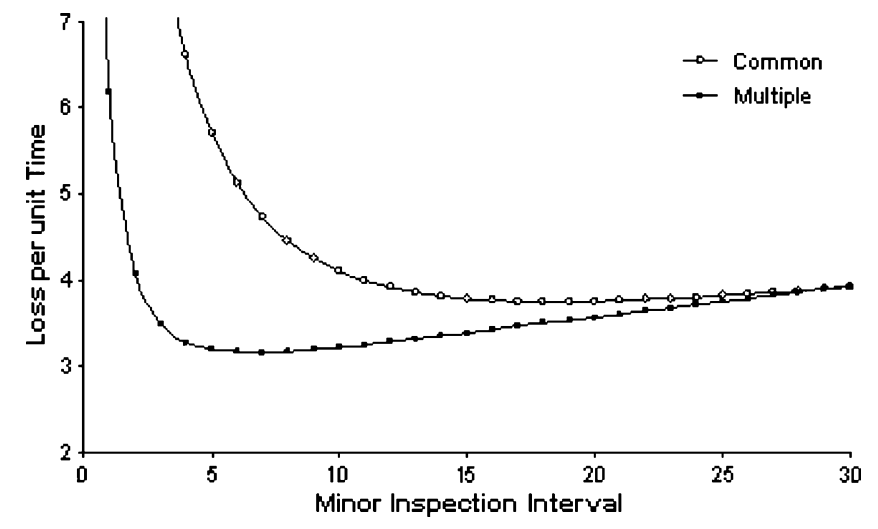

Fig. 4. Comparing the expected loss per unit time against the minor inspection interval for the common and multiple inspection models.

Now, we turn our attention to the more realistic case with multi-level maintenance and service processes over a finite planned operational lifetime. We also present some ideas for incorporating and assessing the impact of technological insertion and upgrading activities on inspection processes..

\section{Finite HORIZON INSPECTION MODELS WITHOUT TECHNOLOGY INSERTIONS}

In the following sections, we consider scheduling applications over a finite time frame, and define $T$ as the planned service life of the asset. We formulate in this section two basic models without technology insertions to be able to compare them with the model we propose in the next section. As there are no technology insertions, the arrival rates for both minor and major defects are constant, and constant intervals are used between consecutive inspections of a given type.

\section{A. A Single Inspection Interval}

In this single inspection case, inspections are scheduled for the system as a whole. The objective function is typically a certain loss or penalty measure. The total expected loss over the service life of the asset includes the summation of the expected numbers of (a) minor failures, (b) minor defects identified and removed at minor inspections, (c) major failures, and (d) major defects identified and rectified at major inspections. If a single inspection on interval $t$ is scheduled for the whole system, where the system has a single average fault arrival rate $\lambda$, and a single delay time pdf for all defects $f(h)$, then the total number of inspection intervals is $\lfloor T / t\rfloor$, where $\lfloor x\rfloor$ is the largest integer less than or equal to $x$. Also, there will be no inspection at the end of the asset service life. It therefore follows that the total expected loss over the planned service life is

$$
\begin{array}{r}
\mathrm{E}[U(t)]=\left\{U_{f} \mathrm{E}\left[N_{f}(t)\right]+U_{s}+U_{d} \mathrm{E}\left[N_{d}(t)\right]\right\}[|T / t|-1] \\
+U_{f} \mathrm{E}\left[N_{f}(T-(\lfloor T / t\rfloor-1) t)\right] .
\end{array}
$$

Note that in the finite horizon case we are interested in the total loss over the planned life rather than the loss per unit time used in the infinite horizon case.
We are interested here in large values of $T$, and comparatively small values of $t$. As such, the expected loss can be approximated as

$$
\begin{aligned}
\mathrm{E}[U(t)] \approx\left\{U_{f} \mathrm{E}\left[N_{f}(t)\right]\right. & \left.+U_{s}+U_{d} \mathrm{E}\left[N_{d}(t)\right]\right\} \\
& \times[(T / t)-1]+U_{f} \mathrm{E}\left[N_{f}(t)\right]
\end{aligned}
$$

which substantially eases the analytical process.

Lemma 3: The sufficient and necessary condition for an optimal $t^{*}$ to minimize (14) is

$$
\frac{U_{s}+\frac{t *^{2}}{T} U_{d} \lambda(1-F(t *))}{\lambda\left(F(t *) t *-\int_{0}^{t *} F(x) d x\right)}=\left(U_{f}-U_{d}\right)
$$

where the loss measures $U_{f}, U_{d}$, and $U_{s}$ are averaged across the whole system.

The proof of Lemma 3 can be shown simply by differentiating (14) with respect to $t$, and then setting $\partial \mathrm{E}[U(t)] / \partial t=0$. Lemma 3 shows that, for an optimal inspection interval to exist, we must have $U_{f}>U_{d}$ because the left-hand side of (14) is always positive due to the fact that, for all $t>0$, we have $1-F(t) \geq 0$, and

$$
F(t) t-\int_{0}^{t} F(x) d x>0
$$

This result is as expected because, if $U_{f} \leq U_{d}$, then there would be absolutely no need for planned inspections just for failurebased maintenance.

Lemma 4: The solution of (15) with respect to $t^{*}$ is unique if

$$
\left(U_{f}-U_{d}\right)>\frac{U_{s}}{\lambda E(H)} .
$$

Proof: Let $g(t)$ denote the left-hand side of (15). Then, when $t=0$, we have $g(0)=\infty$. When $t$ increases, the numerator of $g(t)$ decreases to $U_{s}$ as $F(t)$ rapidly increases to 1 , and $F(t) t-\int_{0}^{t} F(x) d x=E(H)$ as shown in the proof of Lemma 2.

This result shows that $g(t)$ is a decreasing function of $t$ towards

$$
U_{s} /(\lambda E(H)) .
$$

As such, we must have (16) if a solution for (15) exists and is unique because $g(t)$ decreases monotonically.

\section{B. Multiple Inspection Intervals}

If the rates of the arrival of minor and major defects are available with different delay time pdfs and loss measures, the objective function becomes

$$
\begin{aligned}
\mathrm{E}[U(t)]=\sum_{k=1}^{2}\left(\left\{U_{k f}\right.\right. & \left.\mathrm{E}\left[N_{k f}(t)\right]+U_{k s}+U_{k d} \mathrm{E}\left[N_{k d}(t)\right]\right\} \\
& \left.\times[(T / t)-1]+U_{k f} \mathrm{E}\left[N_{k f}(t)\right]\right) .
\end{aligned}
$$

There is still a condition to satisfy if an optimal $t^{*}$ exists to minimize (17), and it is given by the following lemma. 
Lemma 5: The sufficient and necessary condition for an optimal $t^{*}$ to minimize (17) is

$$
\begin{aligned}
\sum_{k=1}^{2}\left(\lambda_{k}\left(U_{k f}-U_{k d}\right)\left(F_{k}(t *) t *-\int_{0}^{t *} F(x) d x\right)\right) \\
=\sum_{k=1}^{2}\left(\frac{t *^{2}}{T} U_{k d} \lambda_{k}\left(1-F_{k}(t *)\right)+U_{k s}\right) .
\end{aligned}
$$

Equation (18) can be obtained in the same way as (15), although the condition $U_{k f}>U_{k d}$ is not strictly required for all defects.

Theorem 1: To minimize the expected loss measure, if separate information about the different defect arrival rates, delay time pdfs, and loss measures are all available, then a multiple inspection scheme at different intervals is at least superior when compared with a common inspection policy, if such optimal intervals exist.

See Appendix for the proof of this theorem.

One common inspection interval for all types of defects is just a special case of the general case of a specific inspection interval for each type of defects, and therefore it would be obvious that the general one will always get at least an equal to or better solution than the special case.

If technological insertions are incorporated, then there may be a reduced rate of the major defect arrivals over time. This change will results in a smaller expected loss than that of the case with no insertions. This result can be used partially to justify the need for technological insertions. However, if the major defect arrival rate is reduced, then a variable inspection interval policy should be employed. This fact is shown in the next section.

\section{DYNAMIC OPTIMIZATION UNDER THE INFLUENCE OF TECHNOLOGICAL INSERTIONS}

To incorporate the impact of technological insertions over time for fixed horizon inspection models, we introduce the following additional assumptions.

- New technology that may lead to component upgrading occurs during major inspections at discrete time epochs, and is assumed to impact upon the arrival rate of major defects.

- The arrival rate for major defects is defined as a function of the time since the last major inspection.

- The interval between minor inspections is constant, but the interval between major inspections, which is influenced by aging and upgrading, varies.

- Major inspections can only occur at the time of a planned minor inspection.

To model the impact of technological insertions over time, $\lambda_{2}\left(t_{i}\right)$ is defined as the major defect arrival rate after the $i$ th major inspection. The inspection process, and the different fault arrival rates are illustrated in Fig. 5.

In fact, technology insertions may also have an impact on minor failures, and the influence may not always be positive, as shown in Fig. 5. These factors can be considered in theory, but the model will be extremely complicated with a variable rate of minor defect arrivals. We leave them to a future study.

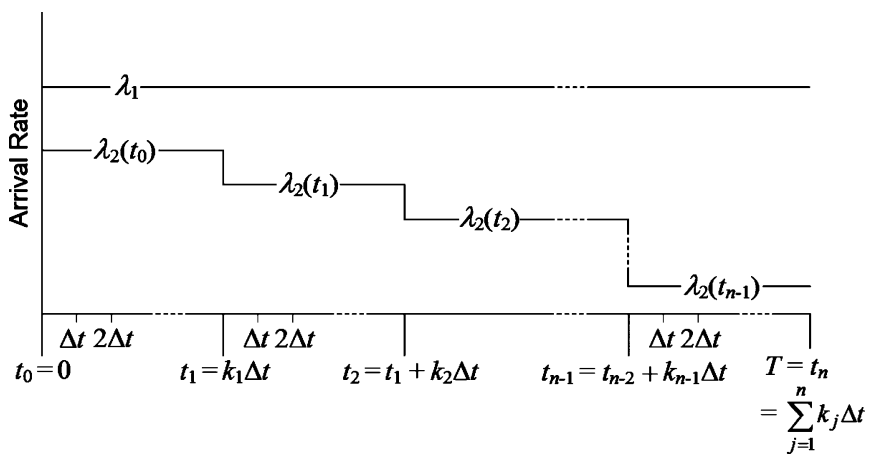

Fig. 5. The inspection process, and the different defect arrival rates over time.

If the major defect arrival rate is influenced by technological insertions over time, we must have a variable inspection scheme for the major inspections. Assuming that there are $n$ major inspections, where $n$ is a decision variable, we have the total expected loss associated with maintenance as

$$
\begin{gathered}
\mathrm{E}\left[U\left(\Delta t, t_{i} ; i=1,2, \ldots, n\right)\right] \\
=\left\{U_{1 f} \mathrm{E}\left[N_{1 f}(\Delta t)\right]+U_{1 s}+U_{1 d} \mathrm{E}\left[N_{1 d}(\Delta t)\right]\right\} \\
\times((T / \Delta t)-1)+U_{1 f} \mathrm{E}\left[N_{1 f}(\Delta t)\right] \\
+\sum_{i=1}^{n-1}\left\{U_{2 f} \mathrm{E}\left[N_{2 f}\left(t_{i}-t_{i-1}\right)\right]+U_{2 s}\right. \\
\left.+U_{2 d} \mathrm{E}\left[N_{2 d}\left(t_{i}-t_{i-1}\right)\right]\right\} \\
+U_{2 f} \mathrm{E}\left[N_{2 f}\left(t_{n}-t_{n-1}\right)\right]
\end{gathered}
$$

where the expected number of minor failures over a minor inspection interval, $\mathrm{E}\left[N_{1 f}(\Delta t)\right]$, is given by (1). The expected number of minor defects removed at a planned minor inspection $\mathrm{E}[N 2 \mathrm{~d}(\Delta t)]$ is given by (3). The expected number of major failures over an interval $\left(t_{i-1}, t_{i}\right)$ between major inspections is defined as $\mathrm{E}\left[\mathrm{N}_{2 f}\left(t i-t_{i-1}\right)\right]$, from (1), but with $\lambda_{2}$ replaced by $\lambda_{2}\left(t_{i-1}\right)$ as

$$
\mathrm{E}\left[N_{2 f}\left(t_{i}-t_{i-1}\right)\right]=\int_{0}^{t_{i}-t_{i-1}} \lambda_{2}\left(t_{i-1}\right) F_{2}(h) d h .
$$

$F_{2}$ is the cumulative delay time distribution for major defects. The expected number of major defects identified and removed at a major inspection at $t_{i}$ is defined as $\mathrm{E}\left[N_{2 d}\left(t_{i}-t_{i-1}\right)\right]$, as given by (3), with $\lambda_{2}$ replaced by $\lambda_{2}\left(t_{i-1}\right)$ :

$$
\mathrm{E}\left[N_{2 d}\left(t_{i}-t_{i-1}\right)\right]=\int_{0}^{t_{i}-t_{i-1}} \lambda_{2}\left(t_{i-1}\right)\left(1-F_{2}(h)\right) d h .
$$

If the loss incurred is measured in terms of cost, then the total expected cost can be compared with the budgetary constraint on the maintenance and service activities. This measure can also be used for contract negotiation if the maintenance service is outsourced.

If the intervals for minor and major inspections are $s$-independent, then the two parts of the right-hand side of (19) can be optimized separately. The optimization of the first part of the right-hand side of (19) is straightforward using Lemma 3. The optimization of the second part is not easy because we have assumed that technological insertions occur at the time of major inspections. As a result, the major inspection interval, and the 
impact of technological insertions are correlated. For reasons of simplicity, we have assumed that major inspections can only occur at the time of a planned minor inspection. This assumption has the managerial advantages of easy planning and optimization, though it may not be globally optimal. The objective function is then

$$
\begin{aligned}
\mathrm{E}[ & \left.U\left(\Delta t, t_{i} ; i=1,2, \ldots, n\right)\right] \\
= & \left\{U_{1 f} \mathrm{E}\left[N_{1 f}(\Delta t)\right]+U_{1 s}+U_{1 d} \mathrm{E}\left[N_{1 d}(\Delta t)\right]\right\} \\
& \times((T / \Delta t)-1)+U_{1 f} \mathrm{E}\left[N_{1 f}(\Delta t)\right] \\
& +\sum_{i=1}^{n-1}\left\{U_{2 f} \mathrm{E}\left[N_{2 f}\left(m_{i} \Delta t\right)\right]+U_{2 s}+U_{2 d} \mathrm{E}\left[N_{2 d}\left(m_{i} \Delta t\right)\right]\right\} \\
& +U_{2 f} \mathrm{E}\left[N_{2 f}\left(m_{n} \Delta t\right)\right](22)
\end{aligned}
$$

where we have

$$
\begin{aligned}
\mathrm{E}\left[N_{2 f}\left(m_{i} \Delta t\right)\right] & =\int_{0}^{m_{i} \Delta t} \lambda_{2}\left(t_{i-1}\right) F_{2}(h) d h \\
& \equiv \int_{0}^{m_{i} \Delta t} \lambda_{2}\left(\sum_{j=1}^{i-1} m_{j} \Delta t\right) F_{2}(h) d h
\end{aligned}
$$

and

$$
\mathrm{E}\left[N_{2 d}\left(m_{i} \Delta t\right)\right]=\int_{0}^{m_{i} \Delta t} \lambda_{2}\left(\sum_{j=1}^{i-1} m_{j} \Delta t\right)\left(1-F_{2}(h)\right) d h
$$

Major defects typically have a longer mean time between failures than minor defects. As such, a longer major inspection interval relative to a shorter minor inspection interval is not an unreasonable assumption, which implies $m_{i} \geq 1$. Because a given major inspection is to be scheduled to coincide with a minor inspection, as we have assumed, then $m_{i}$ must be an integer. This integer constraint is again for managerial consideration; otherwise, $m_{i}$ can be fractional. Due to the fact that the second part of the right-hand side of (22) is a multiple of $\Delta t$, the two parts cannot be optimized separately. In the following, we propose an algorithm to jointly optimize (22) with respect to both $\Delta t$ and $m_{i}$, where $m_{i} \geq 1$.

\section{Optimization Algorithm}

1. For a given $\Delta t$, we have $n=\lfloor T / \Delta t\rfloor$.

2. Calculate

$$
\begin{array}{r}
\mathrm{E}\left[U_{1}(\Delta t)\right]=\left\{U_{1 f} \mathrm{E}\left[N_{1 f}(\Delta t)\right]+U_{1 s}+U_{1 d} \mathrm{E}\left[N_{1 d}(\Delta t)\right]\right\} \\
\times((T / \Delta t)-1)+U_{1 f} \mathrm{E}\left[N_{1 f}(\Delta t)\right] .
\end{array}
$$

(Note that the last term is an approximation because the last minor interval is usually less than $\Delta t$.)

3. Define $f_{i}\left(m_{i} \Delta t\right)$ as the optimal total expected loss due to major failures and inspections given $\Delta t$, and up to the previous major inspection time $t_{i-1}$.

Considering the fact that when $m_{i}=n-\left(t_{i-1} / \Delta t\right)$ there will be no major inspection at $T$, the first step in scheduling the $i$ th major inspection is to determine the next step [see (25) at the bottom of the page].

4.

where, $t_{i-1}=\sum_{j=1}^{i-1} m_{j} \Delta t$.

Dynamic programming (DP) is then used to obtain the recursive equation

$$
f_{i}\left(m_{i} \Delta t\right)=g_{i}\left(m_{i} \Delta t\right) m_{i} \Delta t+f_{i-1}\left(m_{i-1} \Delta t\right)
$$

where $f_{0}\left(m_{0} \Delta t\right)=0, t_{0}=0, t_{i}=t_{i-1}+m_{i} \Delta t$, and $m_{i}=1,2, \ldots, n-\left(t_{i-1} / \Delta t\right)$.

The three elements of the above DP formulation are as follows.

- Stage: $i ; i=1,2, \ldots$

- State: $t_{i-1} ; t_{i-1}=(i-1) \Delta t, \ldots, n \Delta t$, except $t_{0}=0$.

- Decision: $m_{i} ; m_{i}=1,2, \ldots, n-\left(t_{i-1} / \Delta t\right)$.

Note that the number of stages in this DP formulation is unknown, but has an upper bound, $n$. As a consequence, there must be a stopping criteria that terminates the recursive computing. The stopping criteria is: If at stage $c$ we have $m_{c}^{*}=n-\left(t_{m-1} / \Delta t\right)$, then stop. Otherwise, proceed to stage $c+1$.

5.Calculate the total expected loss:

$$
\mathrm{E}\left[U\left(\Delta t, t_{i} ; i=1,2, \ldots, n\right)\right]=\mathrm{E}\left[U_{1}(\Delta t)\right]+f_{n}\left(m_{n} \Delta t\right)
$$

6.The optimal decisions are given as

$$
\begin{aligned}
& \mathrm{E}\left[U\left(\Delta t *, t_{i} * ; i=1,2, \ldots, n\right)\right] \\
& \quad=\min _{\Delta t, m_{1}, m_{2}, \ldots, m_{n}}\left\{\mathrm{E}\left[U\left(\Delta t, t_{i} ; i=1,2, \ldots, n\right)\right]\right\}
\end{aligned}
$$

The proposed dynamic programming algorithm is globally optimal because, for a given $\Delta t$, the space of $m$ is searched to optimize (26). The downside is the lengthy computation time. The above algorithm is under the condition that $\Delta t$ is given. However, $\Delta t$ is also a decision variable to be optimized. In the example below, $\Delta t$ is optimized by enumeration by treating it as a discrete variable. It cannot be optimized analytically in the case of no technology insertions because of the variable number

$$
g_{i}\left(m_{i} \Delta t\right)=\min _{m_{i}}\left\{\begin{array}{ll}
\frac{U_{2 f} \mathrm{E}\left[N_{2 f}\left(m_{i} \Delta t\right)\right]+U_{2 s}+U_{2 d} \mathrm{E}\left[N_{2 d}\left(m_{i} \Delta t\right)\right]}{m_{i} \Delta t} ; & m_{i}=1,2, \ldots,\left(n-\left(t_{i-1} / \Delta t\right)-1\right) \\
\frac{U_{2 f} \mathrm{E}\left[N_{2 f}\left(m_{i} \Delta t\right)\right]}{m_{i} \Delta t} ; & m_{i}=n-\left(t_{i-1} / \Delta t\right)
\end{array}\right\}
$$


of $\Delta t$ within a major inspection interval, and the use of DP. This condition may lead to a suboptimal solution, but it can be close to the true optimal solution depending on the step size used in the enumeration. We admit that this work can be time-consuming if the option for $\Delta t$ is large. However, in practice, even if a non-integer optimal solution is obtained, it has to be rounded to a nearby integer for an actual planning purpose, so our procedure to make $\Delta t$ an integer is justified.

\section{NUMERICAL EXAMPLE}

The two-level maintenance process described for a fixed operational lifetime with the provision for technological upgrading is now demonstrated using some assumed distributional forms, and specified parameters. We illustrate the major inspection decisions as a function of a proposed fixed minor inspection interval, and then demonstrate the process of selection used to ascertain the optimal fixed minor inspection interval, and subsequently the optimal major maintenance inspection sequence.

The constant arrival rate for minor defects is taken to be $\lambda_{1}=0.25$ per month, and the delay time pdf is $f_{1}(h)=\alpha_{1} \exp \left(-\alpha_{1} h\right)$ with parameter $\alpha_{1}=0.1$ per month. The delay time pdf for major defects is $f_{2}(h)=\alpha_{2} \exp \left(-\alpha_{2} h\right)$ with $\alpha_{2}=0.05$ per month.

Now we turn our attention to the modeling of the impact of technological upgrading over time. We assume that asset improvements are manifested in the form of a reducing major defect arrival rate over time. As such, the major defect arrival rate after the $i$ th major inspection is modeled as $\lambda_{2}\left(t_{i}\right)=a+$ $b \exp \left(-c t_{i}\right)$ per month (which is a reducing function of time). For this particular example, we assume that the parameters are $a=0.05, b=0.1$, and $c=0.004$. If $i=0$, and $t^{0}=0$, then we have the starting rate $\lambda_{2}\left(t_{0}\right)=a+b=0.15$ per month, which is smaller than $\lambda_{1}$, as modeled in Sections IV and V. Also, note that, if $c=0$ given $\lambda_{2}\left(t_{i}\right)=a+b$, then the technology insertions have no impact on the defect arrival rate.

The functional form of $\lambda_{2}\left(t_{i}\right)$ demonstrates a situation where the baseline rate is $a$, which cannot be reduced by technological insertions, but $b, c$, and $t_{i}$ control the impact of technological insertions. As $t_{i}$ increases, more and better technological insertions will be performed because of an increased rate of new technology emerging. Therefore, the rate at which $\lambda_{2}\left(t_{i}\right)$ approaches the asymptotic value $a$ increases. For actual case applications, the specification of $\lambda_{2}\left(t_{i}\right)$ will require a substantial amount of data from other assets relating to the impact past modifications had in improving the capability of the asset. Alternatively, technological insertions could also be modeled as affecting (reducing) the expected delay time of a major defect from conception to failure.

The expected numbers of failures and defects removed at an inspection for each defect type are obtained using (1), (3), (25), and (26). In this case, the analytical forms are

$$
\begin{aligned}
\mathrm{E}\left[N_{1 f}(\Delta t)\right]= & \lambda_{1} \Delta t-\left(\lambda_{1} / \alpha_{1}\right)\left(1-e^{-\alpha_{1} \Delta t}\right) \\
\mathrm{E}\left[N_{1 d}(\Delta t)\right]= & \left(\lambda_{1} / \alpha_{1}\right)\left(1-e^{-\alpha_{1} \Delta t}\right) \\
\mathrm{E}\left[N_{2 f}\left(t_{i-1}, t_{i}\right)\right]= & \lambda_{2}\left(t_{i-1}\right)\left(t_{i}-t_{i-1}\right)-\left(\lambda_{2}\left(t_{i-1}\right) / \alpha_{2}\right) \\
& \times\left(1-e^{-\alpha_{2}\left(t_{i}-t_{i-1}\right)}\right)
\end{aligned}
$$

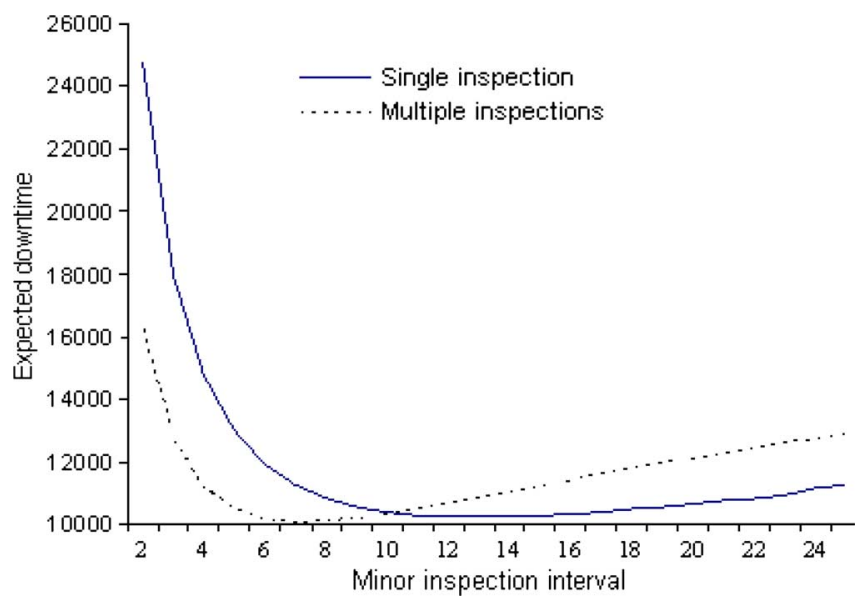

Fig. 6. The total expected downtimes for Case 1, a single inspection interval scheme; and Case 2, a multiple inspection interval scheme when $m=2$.

and

$$
\mathrm{E}\left[N_{2 d}\left(t_{i}\right)\right]=\left(\lambda_{2}\left(t_{i-1}\right) / \alpha_{2}\right)\left(1-e^{-\alpha_{2}\left(t_{i}-t_{i-1}\right)}\right) .
$$

We use downtime values as the loss measures in this example. The assumed downtime information is as follows. For minor defects, the average downtime associated with a minor failure is taken to be $U_{1 f}=100$ minutes, the downtime for a planned minor inspection is $U_{1 s}=40$ minutes, and the downtime for individual defect identification and removal at inspection is $U_{1 d}=10$ minutes. For major defects, the downtime for a failure is $U_{2 f}=500$ minutes, the downtime for a planned major inspection is $U_{2 s}=200$ minutes, and the downtime for individual defect identification and removal is $U_{2 d}=75$ minutes.

We first use the above information to illustrate that a multiple inspection scheme is superior when compared with a single common inspection interval in the case of no technological insertions, which implies that $\lambda_{2}\left(t_{i}\right)=a+b=0.15$. Because both defect arrival rates are constant, the constant inspection intervals are the best option. The optimization process for the multiple inspection is undertaken using enumeration on both $\Delta t$ and $t=m \Delta t$. Using Lemma 3 (15), we have $t^{*}=12.496$, and the total expected downtime is 10249.3 minutes. Jointly optimizing under a multiple inspection interval scheme, we have $\Delta t^{*}=7$, and $m^{*}=2$. This result gives the total expected downtime a value of 10069.1 minutes, which is smaller than the single common inspection scheme of 10249.3 minutes, and also confirms Lemma 4 for this case. Fig. 6 illustrates the total expected downtime in terms of inspection intervals for these two cases. We can also see from Fig. 6 that the optimal result is unique if it exists.

To further illustrate Lemmas 1 and 2, we plot $g(t)$ (the lefthand side of equation (6)) against $t$, which is shown in Fig. 7.

Fig. 7 shows that $g(t)$ is monotone, and decreases against $t$, so a unique solution may exist. From $F_{1}(h)=1-\exp (-\alpha h)$, we have $E(H)=1 / \alpha_{1}$, which gives

$$
U_{1 s} /\left(\lambda_{1} / \alpha_{1}\right)=36.84
$$

Because $U_{1 f}-U_{1 d}=90>36.84$, we can confirm that a unique solution exists. Enumerating against $t$ for the case of minor in- 


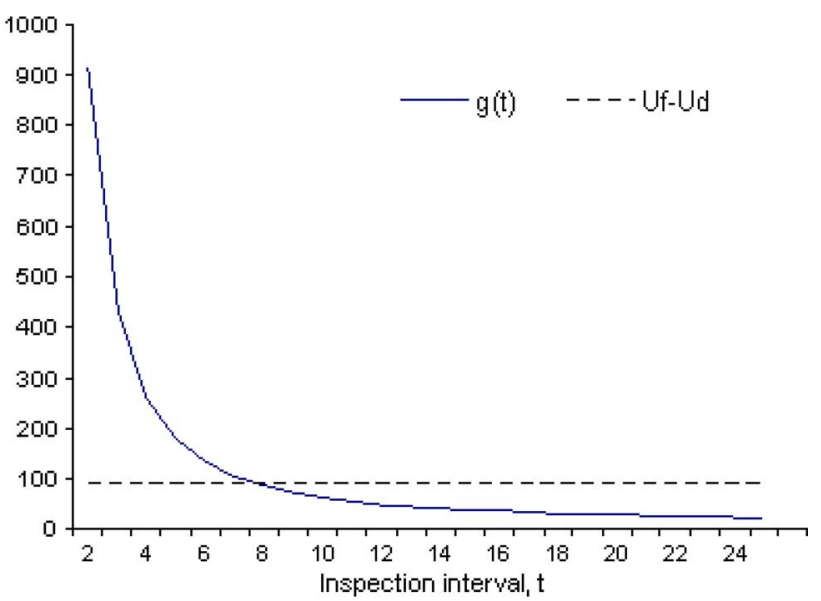

Fig. 7. $g(t)$ against $t$, and the dashed line is $U_{1 f}-U_{1 d}=90$ (using the data from minor inspections only).

TABLE I

SELECTION OF THE OPTIMAL MINOR INSPECTION INTERVAL, AND THE ASSOCIATED SEQUENCE OF MAJOR INSPECTION INTERVALS WHERE * INDICATES THE OPTIMAL SOLUTION

\begin{tabular}{|c|c|c|c|c|c|c|c|c|c|c|c|c|}
\hline \multicolumn{13}{|c|}{$m_{i}{ }^{*}$} \\
\hline$\Delta t$ & $i=1$ & $i=2$ & $i=3$ & $i=4$ & $i=5$ & $i=6$ & $i=7$ & $i=8$ & $i=9$ & $i=10$ & $i=11$ & $\mathrm{E}[U]$ \\
\hline 1 & 14 & 14 & 15 & 15 & 15 & 16 & 16 & 17 & 17 & 41 & - & 14522.5 \\
\hline 2 & 7 & 7 & 7 & 8 & 8 & 8 & 8 & 8 & 9 & 20 & - & 11091.7 \\
\hline 3 & 5 & 5 & 5 & 5 & 5 & 5 & 5 & 6 & 6 & 13 & - & 10050.4 \\
\hline 4 & 4 & 4 & 4 & 4 & 4 & 4 & 4 & 4 & 4 & 9 & - & 9577.1 \\
\hline 5 & 3 & 3 & 3 & 3 & 3 & 3 & 3 & 3 & 3 & 9 & - & 9487.7 \\
\hline 6 & 2 & 2 & 3 & 3 & 3 & 3 & 3 & 3 & 8 & - & - & 9468.5 \\
\hline $7 *$ & 2 & 2 & 2 & 2 & 2 & 2 & 2 & 2 & 2 & 3 & 5 & $9187.9^{*}$ \\
\hline 8 & 2 & 2 & 2 & 2 & 2 & 2 & 2 & 2 & 2 & 5 & - & 9198 \\
\hline 9 & 2 & 2 & 2 & 2 & 2 & 2 & 2 & 2 & 4 & - & - & 9275.7 \\
\hline 10 & 2 & 2 & 2 & 2 & 2 & 2 & 2 & 4 & - & - & - & 9420.3 \\
\hline 12 & 1 & 1 & 1 & 1 & 1 & 1 & 1 & 1 & 1 & 2 & 4 & 9599.7 \\
\hline 15 & 1 & 1 & 1 & 1 & 1 & 1 & 1 & 1 & 1 & 3 & - & 9607.7 \\
\hline 18 & 1 & 1 & 1 & 1 & 1 & 1 & 1 & 1 & 2 & - & - & 9662 \\
\hline 20 & 1 & 1 & 1 & 1 & 1 & 1 & 1 & 2 & - & - & - & 9863.6 \\
\hline
\end{tabular}

spections only, we find that the optimal solution is between 7 and 8 . Solving with respect to $t$, we finally have $t^{*}=7.442$, which is also confirmed in Figs. 6 and 7.

Now we turn to the case with technological insertions. The results of the optimization algorithm presented earlier are given in Table I and Fig. 8 for a required service life of duration $T=$ 180.

Table I illustrates the selection of the optimal minor inspection interval and the associated sequence of major inspection intervals. It shows that the optimal minor inspection interval is 7 months, and the optimal sequence is $m_{i}^{*}=1,2, \ldots, 9$ for all of the major inspections but the last one, which is $m_{10}^{*}=3$. This result implies that one should carry out major inspections at an interval of 14 months until the 9th inspection. The final interval before $T$ should be 21 months.

Fig. 8 illustrates the total expected downtime over the life of the asset for a selection of minor inspection intervals when $m$ is optimized by the dynamic programming algorithm. For continuous $\Delta t$, the optimal minor inspection interval is calculated

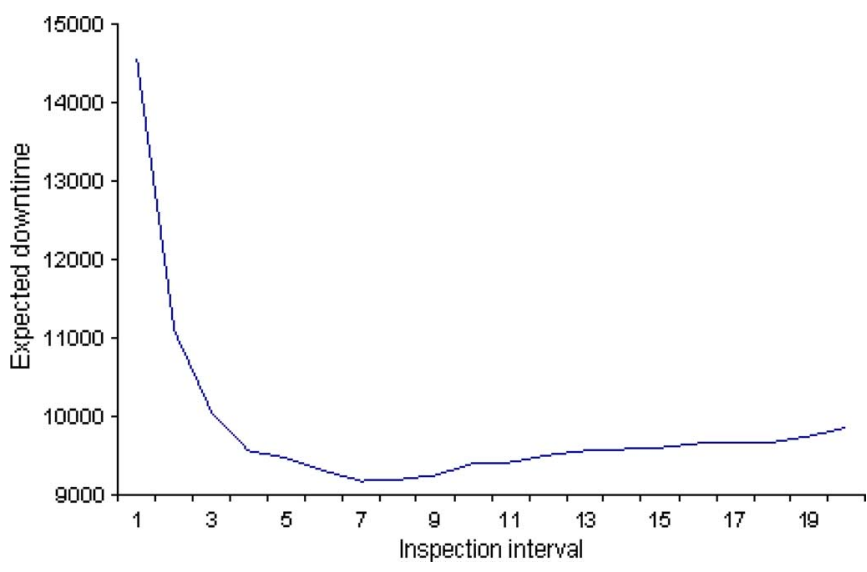

Fig. 8. Total expected downtime for a selection of proposed minor inspection intervals.

as $\Delta t^{*}=7.442$ (obtained by using the optimized $m$ in Table I with $\Delta t=7$, and then re-optimizing $\Delta \mathrm{t}$ ). However, practical limitations mean that it is more likely that the optimal $\Delta t$ will be evaluated as a discrete variable. The optimal discrete interval is found to be $\Delta t^{*}=7$, as shown in Table I. Compared with the result in Fig. 3, we can see that technological insertions reduced the expected downtime to 9188 minutes from 10069. The optimal solutions of minor and major inspections are almost the same for both cases of multiple inspection interval schemes with and without technological insertions, apart from the last major inspection interval. This result is due to the small value for $c$, which models the effect in the reduction of the rate of arrivals of defects due to technological insertions. The slight un-smoothness of the curve in Fig. 8 is due to the use of integer values for $m$, and the DP algorithm.

As illustrated in Table I, the interval between major inspections is observed to increase over time as a consequence of the assumption of technological upgrading activities. This observation is consistent with expectations. And for a practical case application the expected maintenance cost reduction must be weighed against the expected costs associated with the upgrading process. If the cost per unit time is constant and valued at $£ 20$ per unit time, then the total expected cost using the optimal inspection intervals is $9187.9 * 20=£ 187298$ from Table I. This value can then be compared with the budget to see whether the budget is feasible or not.

The cost of technological insertions has not been taken into account explicitly, but can be modeled implicitly by manipulating the values of $b$ and $c$ in $\lambda_{2}\left(t_{i}\right)=a+b \exp \left(-c t_{i}\right)$. Supposing that the total budget for technological insertions is $C_{T}$, then $b$ would be negatively, and c positively, proportional to $C_{T}$. Therefore, when $C_{T}$ is large, then $\lambda_{2}\left(t_{i}\right)=a+b \exp \left(-c t_{i}\right)$ rapidly reduces to $a$, which is the baseline rate (not influenced by $C_{T}$ ). This result will enable us to evaluate the benefit of such technological insertions. Assuming that $b=\beta / C_{T}, c=\alpha C_{T}$, and $C_{T}$ is increased to $£ 11,000$ by $10 \%$ from the current level of $£ 10,000$, then we have the new parameters $b=0.09$, and $c=0.0044$. Using an algorithm similar to the one introduced in Section VI, we have $\Delta t^{*}=5$, and the total downtime is 8996.7 minutes. Still assuming that the downtime cost is constant and valued at $£ 20$ per unit time, then the saved downtime cost is 
$20 *(9187.9-8996.7)=£ 3746$, which is larger than the additional investment in the technological insertions.

The other potential benefits of technological insertions have not been considered yet, such as increased productivity or capability. Although it will be difficult to model them directly, both can be modeled indirectly by altering the cost per unit time. The rationale is that, when productivity or capability increases, then the cost of downtime may increase because more valued outputs are required. For example, a machine that produced 100 items per unit time before its capacity was increased to 200 items per unit time by inserting new technology would have double the cost of downtime due to the loss of production. This result will be validated using a case study in the future.

\section{CONCLUSION}

Using the delay time concept, we modeled a two-level inspection problem subject to technological insertions. This model can be used for optimizing both inspection intervals and evaluating the impact of technological insertions upon the asset performance in terms of cost or downtime. It can also be used as a tool for the relationship analysis between technology insertions and inspection schedules, and for negotiation when the maintenance is outsourced. The impact of technological insertions has been modeled by changing the rate of arrival of major defects, but the rate of arrival of minor defects is assumed to be constant. We have given several lemmas that relate to the properties of the model developed. We showed that a multiple inspection interval scheme is always better than a single common inspection scheme if the failures mechanisms and utilities can be modeled differently. We also show that a variable inspection interval is best for major inspections if the rate of arrival of major defects is a function of the time since the last major inspection, and is influenced by technological insertions.

The numerical example confirmed our findings in the theoretical development, and demonstrated that the optimal major inspection intervals are in order of an increasing pattern, which is due to the increased impact of technological insertions in reducing the rate of arrival of major defects. Many extensions can be made such that the technology insertions can also alter the delay time of both minor and major defects. The arrival rate of minor defects can also be influenced by technology insertions. Although only two levels of inspection are considered in this paper, the modeling principle can be extended to more than two levels. However, this work will require substantial effort in optimization. We also briefly discussed the cost of technology insertions upon the rate of the arrival of major defects, and the influence on productivity and capability. Many other possible ways of manipulating the impact of technology insertions can be explored, and these ways require further research and case validation.

\section{APPENDIX A}

\section{A. Proof of Theorem 1}

The objective function of the expected loss function is the sum of two separate functions. Each is in the form of (14), but with different decision variables, say $\Delta t$ and $t$. We also use $t^{\prime}$ to represent the common inspection interval if (14) is used. The objective functions become as follows.

1) With different inspection intervals:

$$
\begin{aligned}
\mathrm{E}[U(\Delta t, t)]= & \left\{U_{1 f} \mathrm{E}\left[N_{1 f}(\Delta t)\right]+U_{1 s}+U_{1 d} \mathrm{E}\left[N_{1 d}(\Delta t)\right]\right\} \\
& \times[T / \Delta t-1]+U_{1 f} \mathrm{E}\left[N_{1 f}(\Delta t)\right] \\
& +\left\{U_{2 f} \mathrm{E}\left[N_{2 f}(t)\right]+U_{2 s}+U_{2 d} \mathrm{E}\left[N_{2 d}(t)\right]\right\} \\
& \times[T / t-1]+U_{2 f} \mathrm{E}\left[N_{2 f}(t)\right] \\
= & \mathrm{E}\left[U_{1}(\Delta t)\right]+\mathrm{E}\left[U_{2}(t)\right] .
\end{aligned}
$$

2) With one common interval:

$$
\begin{aligned}
\mathrm{E}\left[U\left(t^{\prime}\right)\right]= & \left\{U_{1 f} \mathrm{E}\left[N_{1 f}\left(t^{\prime}\right)\right]+U_{1 s}+U_{1 d} \mathrm{E}\left[N_{1 d}\left(t^{\prime}\right)\right]\right\} \\
& \times\left[T / t^{\prime}-1\right]+U_{1 f} \mathrm{E}\left[N_{1 f}\left(t^{\prime}\right)\right] \\
& +\left\{U_{2 f} \mathrm{E}\left[N_{2 f}\left(t^{\prime}\right)\right]+U_{2 s}+U_{2 d} \mathrm{E}\left[N_{2 d}\left(t^{\prime}\right)\right]\right\} \\
& \times\left[T / t^{\prime}-1\right]+U_{2 f} \mathrm{E}\left[N_{2 f}\left(t^{\prime}\right)\right] \\
= & \mathrm{E}\left[U_{1}\left(t^{\prime}\right)\right]+\mathrm{E}\left[U_{2}\left(t^{\prime}\right)\right] .
\end{aligned}
$$

Suppose $\Delta t^{*}, t^{*}$, and $t^{\prime *}$ are optimized intervals. If they exist, then we may reasonably assume that $\Delta t^{*} \neq t^{*} \neq t^{\prime *}$. Because both $\mathrm{E}\left[U_{1}\left(\Delta t^{*}\right)\right] \leq \mathrm{E}\left[U_{1}\left(t^{\prime} *\right)\right]$ and $\mathrm{E}\left[U_{2}\left(t^{*}\right)\right] \leq \mathrm{E}\left[U_{2}\left(t^{\prime} *\right)\right]$ by definition, then we have

$$
\mathrm{E}\left[U_{1}\left(\Delta t^{*}\right)\right]+\mathrm{E}\left[U_{2}\left(t^{*}\right)\right] \leq \mathrm{E} \leq\left[U\left(t^{*}\right)\right] .
$$

\section{REFERENCES}

[1] R. P. Nicola and R. Dekker, Optimal Maintenance of Multi-Component Systems: A Review, D. N. P Murthy and A. K. S. Kobbacy, Eds. Amsterdam: Complex System Maintenance Handbook, Springer, 2008.

[2] C. Sriram and A. Hagham, "An optimization model for aircraft maintenance scheduling and re-assignment," Transportation Research Part A: Policy and Practice, vol. 37, no. 1, pp. 29-48, 2003.

[3] J. V. Bukowski, "Modeling and analyzing the effects of periodic inspection on the performance of safety-critical systems," IEEE Trans. Reliability, vol. 50, no. 3, pp. 321-329, 2001.

[4] G. Waeyenbergh and L. Pintelon, "A framework for maintenance concept development," International Journal of Production Economics, vol. 77, pp. 299-313, 2002.

[5] W. Wang, "A model of multiple nested inspections at different intervals," Computers \& Operations Research, vol. 27, no. 6, pp. 539-558, 2000.

[6] W. Wang, "An inspection model for a process with two types of inspections and repairs," Reliability Engineering and System Safety, vol. 94, pp. 526-533, 2009.

[7] C. I. V. Kerr, R. Phaal, and D. R. Probert, "Technology insertion in the defence industry: A primer," Proc. IMechE Part B: J. Engineering Manufacture, vol. 222, no. 8, pp. 1009-1023, 2008.

[8] G. Strong, "Technology insertion-a worldwide perspective," J. Defence Sci., vol. 9, no. 3, pp. 114-121, 2004.

[9] T. Dowling, "Technology insertion and obsolescence," J. Defence Sci., vol. 9, no. 3, pp. 151-155, 2004.

[10] A. H. Christer and W. M. Waller, "Reducing production downtime using delay time analysis," J. Opl. Res. Soc., vol. 35, pp. 499-512, 1984.

[11] D. I. Cho and M. Parlar, "A survey of maintenance models of multiunit systems," European Journal of Operational Research, vol. 51, pp. $1-23,1991$.

[12] M. M. Hosseini, R. M. Kerr, and R. B. Randall, "An inspection mode with minimal and major maintenance for a system with deterioration and Poisson failures," IEEE Trans. Reliability, vol. 49, no. 1, pp. 88-98, 2000.

[13] W. Li and H. Pham, "An inspection-maintenance model for systems with multiple competing processes," IEEE Trans. Reliability, vol. 54 , pp. 318-327, 2005. 
[14] L. R. Cui, H. T. Loh, and M. Xie, "Sequential inspection strategy for multiple systems under availability requirement," European Journal of Operational Research, vol. 155, no. 1, pp. 170-177, 2004.

[15] A. H. Christer, "Developments in delay time analysis for modelling plant maintenance," J. Opl. Res. Soc., vol. 50, pp. 1120-1137, 1999.

[16] R. D. Baker and W. Wang, "Determining the delay time distribution of faults in repairable machinery from failure data," IMA J. Maths. Applied in Bus. and Ind., vol. 3, pp. 259-282, 1991.

[17] A. H. Christer, W. Wang, R. D. Baker, and J. Sharp, "Modelling maintenance practice of production plant using the delay-time concept," IMA J Management Maths, vol. 6, pp. 67-83, 1995.

[18] W. Wang and A. H. Christer, "Solution algorithms for a nonhomogeneous multi-component inspection model," Computers and Operations Research, vol. 30, no. 1, pp. 190-34, 2003.

[19] W. Wang, Delay Time Modelling, in Complex System Maintenance Handbook, P. Murthay and A. K. H. Kobbacy, Eds. : Springer, 2008, pp. $345-370$.

[20] A. Akbarov, W. Wang, and A. H. Christer, "Problem identification in the frame of maintenance modeling: A case study," International Journal of Production Research, vol. 46, no. 4, pp. 1031-1046, 2008.

[21] A. H. Christer, W. Wang, J. M. Sharp, and R. D. Baker, "A stochastic modeling problem of high-tech steel production plant," in Stochastic Modelling in Innovative Manufacturing, Lecture Notes in Economics and Mathematical Systems, A. H Christer, S. Osaki, and L. C. Thomas, Eds. Berlin: Springer, 1997, pp. 196-214.

[22] B. Jones, I. Jenkinson, and J. Wang, "Methodology of using delay time analysis for a manufacturing industry," Reliability Engineering and System Safety, vol. 94, pp. 111-124, 2009.

[23] A. Pillay, J. Wang, A. D. Wall, and T. Ruxton, "A maintenance study of fishing vessel equipment using delay-time analysis," Journal of Quality in Maintenance Engineering, vol. 7, no. 2, pp. 118-128, 2001.

Wenbin Wang is a Professor and the Dean of Dongling School of Economics and Management at University of Science and Technology Beijing (USTB), China. Prior to joining USTB in 2011, he was professor at Salford Business School, University of Salford, U.K. Professor Wang received his B.Sc. (Harbin, China) in Mechanical Engineering in 1981, M.Sc. (Xi'an, China) in Operations Management in 1984, and Ph.D. in OR and Applied Statistics from the University of Salford in 1992. He has over 25 years experience in OR modeling in general, and maintenance and reliability modeling in particular. He has published over 150 research papers in maintenance modeling, and related areas.
Matthew J. Carr is a Research Associate within the School of Medicine at The University of Manchester, U.K. Prior to joining the university of Manchester, he was at the Salford Business School, University of Salford, U.K.

Dr Carr received his B.Sc. in Business Operation and Control in 2001, M.Sc. in Operational Research and Applied Statistics in 2002, and Ph.D. in Mathematics from the University of Salford in 2006.

Tommy W. S. Chow (M'93-SM'03) received the B.Sc. (1st Hons) degree, and the Ph.D. degree from the Department of Electrical and Electronic Engineering, University of Sunderland, U.K. He is currently a Professor in the Department of Electronic Engineering at the City University of Hong Kong. He is an author and co-author of over 150 technical Journal articles related to his research, 5 book chapters, and 1 book. His main research has been in the area of neural networks, machine learning, pattern recognition, and fault diagnosis. He received the Best Paper Award at the 2002 IEEE Industrial Electronics Society Annual meeting in Seville, Spain.

Michael G. Pecht (S'78-M'83-SM'90-F'92) is currently a Visiting Professor in Electronic Engineering at City University in Hong Kong. He has an M.S. in Electrical Engineering, and an M.S. and Ph.D. in Engineering Mechanics from the University of Wisconsin at Madison. He is the founder of CALCE (Center for Advanced Life Cycle Engineering) at the University of Maryland, which is funded by over 150 of the world leading electronics companies at more than US\$dollar\$6M/year. He is also a Chair Professor in Mechanical Engineering, and a Professor in Applied Mathematics at the University of Maryland. He has written more than twenty books, and over 400 technical articles. He consults for 22 major international companies, providing expertise in strategic planning, design, test, intellectual property, and risk assessment of electronic products. 